\title{
Hierarquia de Valores Transculturais e Brasileiros ${ }^{1}$
}

\author{
Alvaro Tamayo \\ Universidade de Brasília
}

\begin{abstract}
RESUMO - Foi estabelecida a hierarquia de 56 valores transculturais e quatro valores característicos da cultura brasileira com uma amostra de 419 sujeitos, de ambos os sexos, constituída por professores de escola secundária e estudantes universitários. A hierarquia foi estabelecida tanto no plano dos valores individuais como no dos tipos motivacionais de valores. As diferenças entre os subgrupos da amostra foram verificadas em termos do nível dos tipos motivacionais e da estrutura bidimensional dos valores. A hierarquia apresentou cinco níveis bem distintos de valores. Do ponto de vista motivacional, os valores de autodeterminação ocuparam o primeiro lugar. As mulheres enfatizaram mais do que os homens os valores a serviço de interesses coletivos e de autotranscendência de seus interesses egoístas em benefício do bem-estar dos outros. Os estudantes universitários salientaram mais do que os professores os valores relativos à autopromoção e à abertura à mudança.
\end{abstract}

Palavras-chave: valores; gênero; valores individuais; tipos motivacionais de valores.

\section{Hierarchy of Cross-Cultural and Brazilian Values}

\begin{abstract}
The hierarchy of 56 cross-cultural values and four characteristic values of the Brazilian culture were established with a sample of 419 subjects, of both sexes, constituted by high school teachers and college students. The hierarchy was established so much in the plan of the individual values as in the one of the motivational values types. The differences among the subgroups of the sample were verified in terms of the level of the motivational types and of the bi-dimensional structure of the values. The hierarchy presented five levels very different from values. From the motivational point of view, the selfdetermination values occupied the first place. The women emphasized the values more than the men to service of collective interests and of self-transcendence of their selfish interests in benefit of the well-being of the other ones. The college students pointed out the relative values more than the teachers to the self-promotion and the opening to the change.
\end{abstract}

Key words: values; gender; individual values; motivational values types.

Um sistema de valores, segundo Rokeach (1968/69, p. 551), é "nada mais do que uma disposição hierárquica de valores, uma classificação ordenada de valores ao longo de um contínuo de importância". Os valores implicam necessariamente uma preferência, uma distinção entre o importante e o secundário, entre o que tem valor e o que não tem. Assim, a essência mesma dos valores parece ser permitir a sua hierarquização. A organização hierárquica de valores pressupõe que o indivíduo não se relacione com o mundo físico e social como um observador que assiste a um espetáculo, mas com um ator que participa, que toma partido, que se envolve nele. A idéia de graus de valor, de uma escala de valores ao longo de um contínuo de importância, encontra a sua base na relação dos valores com o tempo, elemento fundamental para o seu desenvolvimento, com o desejo, cuja multiplicidade e veemência impõem uma ordem de primazia, e com o esforço realizado pelo indivíduo para a obtenção das metas transituacionais que constituem os valores. A originalidade do conceito de hierarquia de valores é que ele permite a comparação de indivíduos, grupos sociais e culturas não somente em termos de cada um dos valores mas, particularmente, no plano das prioridades axiológicas.

1 Reedição de texto publicado em 1994 , no volume 10 número 2, na revista Psicologia: Teoria e Pesquisa (pp. 269-286).

\section{Estudos empíricos}

Numerosos pesquisadores, particularmente nos Estados Unidos, têm procurado estabelecer a hierarquia de valores da sociedade. Vários estudos têm sido realizados nesse sentido, inclusive com amostras nacionais (Ball-Rokeach, Rokeach \& Grube, 1984; Rokeach, 1971, 1974). Rokeach e Ball-Rokeach (1989) compararam a hierarquia de valores da sociedade estado-unidense, obtida em momentos diferentes, com amostras nacionais e representativas. Os dois primeiros levantamentos foram realizados pela National Opinion Research Center em $1968(N=1409)$ e $1971(N=1430)$ e analisados comparativamente por Rokeach em 1974. Posteriormente, o Institute for Social Search da Universidade de Michigan realizou dois novos levantamentos em 1974 e 1981, cada vez com uma amostra de 933 sujeitos. Nesses dois últimos levantamentos foram avaliados somente os valores terminais. Ao comparar os resultados obtidos em 1968 com os de 1981, Inglehart (1985) concluiu que a hierarquia é bastante consistente através do tempo. Os valores classificados nos seis primeiros lugares em 1981, família, paz, liberdade, auto-respeito, felicidade e sabedoria, foram os mesmos que em 1968. O mesmo tipo de convergência foi observado em relação aos valores colocados nos seis últimos lugares da hierarquia: reconhecimento social, prazer, beleza, vida excitante, amor maduro e harmonia interior. 


\section{A. Tamayo}

Rokeach e Ball-Rokeach (1989) observaram uma mudança dramática na hierarquia, nesse mesmo período, nos valores com importância intermediária. Por exemplo, em 1971 o valor "igualdade" passou do sétimo para o quarto lugar e em 1974 desceu para o $12^{\mathrm{a}}$ lugar. Essa diminuição da importância dada à igualdade foi acompanhada de uma ascensão na hierarquia de alguns valores pessoais, tais como "vida confortável" e "auto-realização".

No Brasil, Pereira (1986) estabeleceu a hierarquia dos valores terminais ou instrumentais da escala de Rokeach com uma amostra constituída por 680 sujeitos. Os valores terminais mais importantes foram "liberdade", "equilíbrio interior", "auto-respeito", "felicidade" e "segurança da família". O extremo inferior da hierarquia foi ocupado pelos valores "salvação", "mundo de beleza", "vida excitante", "prazer" e "segurança nacional". Em relação aos valores instrumentais, os cinco mais importantes foram: "honesto", "responsável", "amoroso", "capaz" e "alegre"; e os cinco menos importantes: "tolerante", "obediente", "prestativo", "polido" e "ambicioso".

Vários autores (Braithwaite \& Law, 1985; Gorsuch, 1970; Keats \& Keats, 1974; Kitwood \& Smithers, 1975; Lynn, 1974) têm criticado o modelo ordinal da escala de Rokeach e a sua estratégia de classificação dos valores. Segundo os autores mencionados, o pesquisador não tem certeza se o sujeito, ao responder, considera a série total de valores ou somente alguns deles em detrimento dos outros. Tamayo (1988) estudou a hierarquia de valores terminais de Rokeach tentando contornar as dificuldades acima mencionadas. Com esse fim, utilizou uma escala de cinco pontos mediante a qual os sujeitos deviam avaliar a importância que cada um dos valores tinha para eles como princípios orientadores da sua vida. A amostra foi constituída por 1.258 sujeitos, sendo 614 homens e 644 mulheres, 591 residentes em Brasília e 667 em Fortaleza. Do ponto de vista da idade, a amostra foi composta por 413 adolescentes, com idade média de 16,5 anos e cursando os três últimos anos de escola; 408 pósadolescentes, com idade média de 22 anos, todos estudantes universitários e 437 adultos, idade média 35 anos, todos portadores de diploma universitário.

Para elaborar a hierarquia de valores, Tamayo (1988) calculou o valor crítico necessário para se obter uma diferença significativa $(\mathrm{p}, 01)$ entre duas médias. Esse valor foi de 0,10 . Ele foi utilizado como intervalo crítico para dividir a escala vertical em vários segmentos iguais. Nos espaços assim delimitados foram situados os valores, cada um deles de acordo com o seu escore médio. Ao distribuir os valores na escala vertical formaram-se cinco conjuntos, separados nitidamente por espaços vazios, delineando assim cinco níveis hierárquicos de valores. No primeiro nível situaram-se os seguintes valores: "liberdade", "saúde", "felicidade", "paz", "auto-respeito", "equilíbrio interior", "honestidade", "amor", "família", "solidariedade". No segundo nível "respeito à natureza", "sabedoria", "fidelidade" e "igualdade"; no terceiro "sexo" e "prazer"; no quarto "conforto", "dinheiro", "vida estimulante", "patriotismo" e "religião"; no quinto e último nível "beleza" e "reconhecimento social".

A comparação entre as hierarquias aqui discutidas deve ser feita com cautela, porque os estudos realizados com amostras estado-unidenses (Ball-Rokeach \& col., 1984; Inglehart, 1985; Rokeach \& Ball-Rokeach,1989; Rokeach, 1971, 1974) e o de
Pereira (1986) com amostra brasileira são de tipo ordinal, ao passo que o de Tamayo (1988) baseia-se numa escala de intervalos. Nos dois estudos realizados com amostras brasileiras observa-se uma concordância geral na distribuição hierárquica dos valores. A diferença mais marcante parece ser no plano do prazer, penúltimo para a amostra estudada por Pereira (1986) e intermediário no estudo de Tamayo (1988). Essa diferença talvez possa ser atribuída a diferenças culturais entre as duas amostras, já que a primeira foi constituída por sujeitos de Uberlândia e a segunda por sujeitos de Brasília e de Fortaleza. Ao comparar as hierarquias de valores obtidas com amostras brasileiras com as dos Estados Unidos observa-se também uma convergência na disposição geral dos valores. As diferenças mais marcantes são com respeito aos seguintes valores: 1) equilíbrio interior, classificado pelos sujeitos dos Estados Unidos entre os seis últimos e pelas amostras brasileiras entre os sete primeiros; 2 ) amor maduro, situado entre os valores do primeiro nível hierárquico numa das amostras brasileiras (Tamayo, 1988) e entre os seis últimos para os sujeitos dos Estados Unidos; 3) amizade e prazer certamente mais importantes para os sujeitos brasileiros (Tamayo, 1988) do que para os estado-unidenses.

\section{Hierarquia de tipos motivacionais}

Com base nos resultados de pesquisas realizadas recentemente sobre a estrutura motivacional dos valores (Schwartz, 1992; Schwartz \& Bilsky, 1987, 1990; Tamayo \& Schwartz, 1993), a hierarquia axiológica pode ser estabelecida também a partir dos tipos motivacionais. Operacionalmente, um tipo motivacional é um fator composto por diversos valores que apresentam similaridade do ponto de vista do conteúdo motivacional. Por meio do método da Smallest Space Analysis, SSA (Canter, 1985; Guttman, 1968), os valores são representados como pontos no espaço multidimensional, de tal forma que as distâncias entre eles expressem as relações empíricas entre os valores tal como elas foram mensuradas pelas correlações entre seus graus de importância, de acordo com as respostas dos sujeitos. Desse modo, diversos tipos motivacionais têm sido identificados tanto no Brasil (Tamayo \& Schwartz, 1993) como em várias outras culturas (Schwartz, 1992).

Os tipos motivacionais já verificados empiricamente (Figura 1) são os seguintes: 1) Hedonismo: A gratificação de necessidades físicas é transformada em valores socialmente reconhecidos. A meta motivacional desse grupo de valores é o prazer e a gratificação sensual. 2) Auto-realização: Sua meta é o sucesso pessoal obtido por uma demonstração de competência que, geralmente, leva ao reconhecimento social. 3) Poder social: O funcionamento da sociedade parece exigir algum sistema de estratificação de função e de responsabilidade. Dessa forma, surgem no grupo as relações de dominação e submissão. Para justificar esse fato da vida social, o poder é transformado em valor. Necessidades individuais de dominação e de controle podem também ser transformadas em valores relativos ao poder (Schwarzt, 1992). A meta desse tipo de valores é a procura de status social, prestígio e controle sobre pessoas e recursos. 4) Autodeterminação: Os valores de autodeterminação procuram a independência de pensamento, de ação, de opção. 5) Conformidade: Sua meta motivacional é o controle de impulsos e do próprio comportamento em conformidade com as expectativas sociais. 6) Benevolência: A meta motivacional é o interesse e a preocupação com o 
bem-estar das pessoas íntimas. 7) Segurança: A meta dos valores desse tipo é a integridade pessoal e de pessoas e grupos de identificação, assim como a estabilidade da sociedade e de si mesmo. 8) Tradição: Todas as sociedades desenvolvem usos, costumes, práticas e símbolos que representam o seu destino e a sua experiência comum. A tradição é algo que brota da comunidade e que se transforma em símbolo da sua própria sobrevivência (Durkheim, 1954). A meta motivacional dos valores relativos à tradição é o respeito e a aceitação dos ideais e costumes da sua sociedade. 9) Estimulação: Necessidade de excitação, novidade e mudança, a fim de poder manter um nível satisfatório de funcionamento. 10) Filantropia: A meta motivacional desse grupo de valores é a procura do bem-estar de todos. Schwartz denomina esse tipo motivacional "universalism".

\section{Estrutura dinâmica dos tipos motivacionais}

Os tipos motivacionais de valores relacionam-se entre si de forma dinâmica. Os valores ao serviço de interesses individuais são opostos àqueles que servem interesses coletivos (Schwartz, 1992; Tamayo \& Schwartz, 1993). Dessa forma, postulam-se dois tipos básicos de relacionamento entre eles: de compatibilidade e de conflito. Na Figura 1 representa-se a estrutura dinâmica dos tipos motivacionais. $\mathrm{O}$ arranjo das áreas correspondentes aos tipos motivacionais refletem as relações dinâmicas existentes entre eles: compatibilidade para os tipos adjacentes e conflito para os opostos (Schwartz, 1992).

$\mathrm{Na}$ estrutura motivacional, representada na Figura 1, os cinco tipos de valores que expressam interesses individuais (autodeterminação, estimulação, hedonismo, realização e poder social) ocupam uma área contígua que é oposta àquela reservada aos três conjuntos de valores que expressam primariamente interesses coletivos (benevolência, tradição e conformidade). Os tipos motivacionais segurança e filantropia, constituídos por valores que expressam interesses tanto individuais como coletivos, situam-se nas fronteiras dessas duas áreas. Schwartz e Bilsky $(1987,1990)$ postulam compatibilidade entre os tipos de valores adjacentes (por exemplo, estimulação e hedonismo, tradição e conformidade) e conflito entre os tipos de valores situados em direções opostas (exemplo: estimulação e conformidade, hedonismo e tradição). A busca simultânea de valores pertencentes a áreas adjacentes é compatível porque esse tipo de valor está a serviço de um mesmo interesse. Assim, por exemplo, o poder social e a realização ambos procuram a superioridade e a estima social; a tradição e a conformidade perseguem ambas a submissão e o autocontrole. Pelo contrário, a busca simultânea de valores situados em áreas opostas na estrutura motivacional da Figura 1 é conflitiva porque eles estão a serviço de interesses opostos. A preservação da estabilidade e das práticas tradicionais, presente nos valores do tipo tradição, é conflitiva com a procura de mudança e de novidade, que é o núcleo dos valores do tipo estimulação.

\section{Estrutura bidimensional}

A relação estrutural básica entre os valores e entre os tipos motivacionais por eles constituídos pode ser sintetizada por duas dimensões bipolares já verificadas empiricamente tanto no Brasil (Tamayo \& Schwartz, 1993) como no exterior (Schwartz, 1992). A primeira, abertura à mudança versus conservação, ordena os valores com base na motivação da pessoa a seguir os seus próprios interesses intelectuais e afetivos por caminhos incertos e ambíguos, em oposição à tendência a preservar o status $q u o$ e a segurança que ele gera no relacionamento com os outros e com as instituições. Teoricamente, situam-se num dos pólos desse eixo os valores relativos à estimulação e à autodeterminação e, no outro, os referentes à "segurança", à "conformidade" e à "tradição" (Figura 2). A segunda dimensão, "auto- promoção versus auto-transcendência”, apresenta, num dos extremos, os valores relativos aos tipos motivacionais "poder", "auto-realização" e "hedonismo" e, no outro, os valores que integram a "filantropia" e a "benevolência". Esse eixo ordena os valores com base na motivação da pessoa para promover os seus próprios interesses, mesmo às custas dos outros, em oposição a transcender as suas preocupações egoístas e promover o bem-estar dos outros e da natureza.

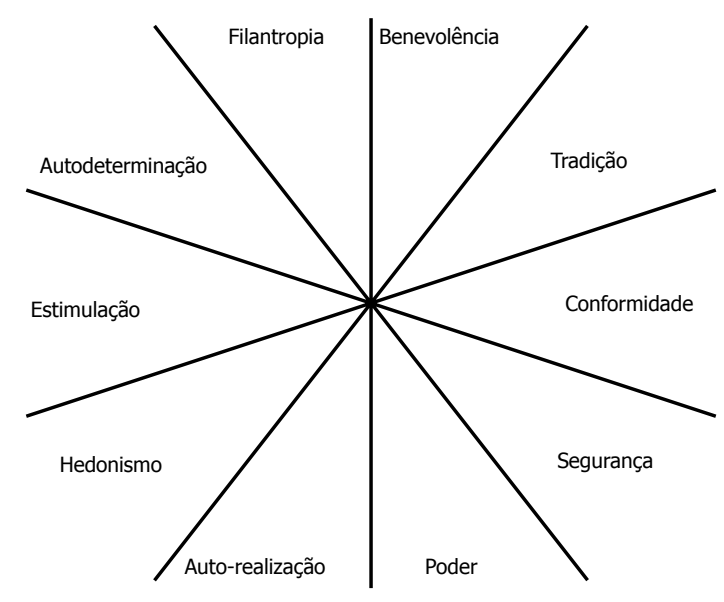

Figura 1. Estrutura motivacional dos valores.

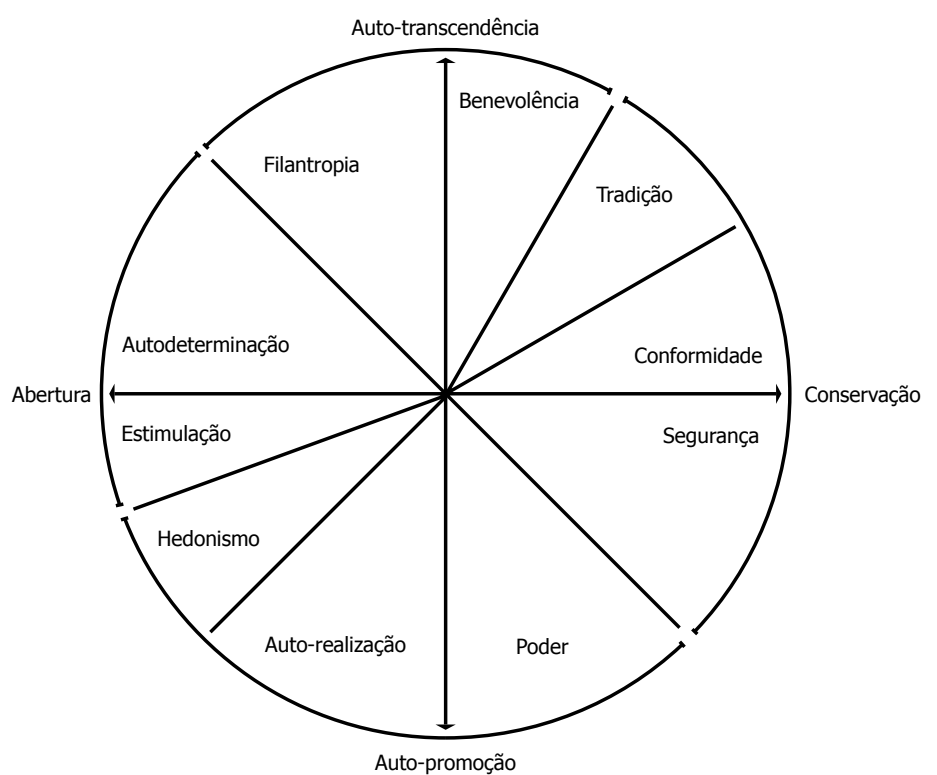

Figura 2. Estrutura bidimensional dos valores. 


\section{A. Tamayo}

Até onde se sabe, ainda não foi publicado estudo baseado nos índices de importância dos tipos motivacionais e na relação estrutural definida pelas dimensões de autotranscendência $v s$ autopromoção e abertura à mudança $v s$ conservação, mas não cabe dúvida de que esse método permite estabelecer comparações intergrupo e intragrupo que terão uma maior significação psicológica do que comparações realizadas a partir dos valores individuais e da sua organização hierárquica.

\section{Mensuração dos valores}

A grande maioria da pesquisa realizada no ocidente utiliza como instrumento de medida a escala de Rokeach. No Brasil, os dois estudos sobre a hierarquia de valores estão baseados também nos 36 valores da escala de Rokeach. Até que ponto os estudos realizados a partir dessa amostra de valores expressam a estrutura axiológica de uma sociedade? Vários autores (Bond, 1988; Braithwaite \& Law, 1985) têm manifestado preocupação com a representatividade dos valores da escala de Rokeach. Será que eles constituem uma amostra suficientemente representativa dos mais importantes valores humanos? Rokeach (1973) insiste que os 36 valores da sua escala são razoavelmente representativos do universo dos valores. Braithwafte e Law (1985) concluíram, após pesquisa empírica, que a escala de valores de Rokeach cobre adequadamente a área multidimensional dos valores humanos. Bond (1988) mostrou que alguns dos valores contidos na Chinese Value Survey (Chinese Culture Connection, 1987) complementam adequadamente a amostra axiológica da escala de Rokeach. Schwartz (1992), numa pesquisa multicultural realizada recentemente, utilizou uma lista de valores que parece ser mais representativa que a de Rokoach já que ela foi obtida a partir de instrumentos de medida elaborados em diversas culturas (Braithewaite \& Law, 1985; Chinese Culture Connection, 1987; Hofstede, 1980; Munro, 1985; Rokeach, 1973).

Embora a escala de Rokeach e, ainda mais, a de Schwartz, representem um núcleo dos mais importantes valores humanos, parece indicado e necessário pesquisar a existência de possíveis valores peculiares, ou mesmo específicos, à cultura para a qual se pretende estabelecer a hierarquia de valores e identificar a sua estrutura motivacional. $\mathrm{O}$ autor identificou quatro valores que parecem ser característicos da cultura brasileira. Utilizando um dos métodos apresentados por Rokeach (1979) para identificar os valores de uma sociedade, Tamayo entrevistou individualmente 20 educadores e ministros de diversas afiliações religiosas. Os sujeitos foram questionados sobre a existência de um ou mais valores que pudessem ser considerados como específicos aos brasileiros. A convergência entre os sujeitos foi surpreendente e, dessa forma, foi fácil identificar quatro valores que parecem ser peculiares aos brasileiros. Esses valores foram: 1) "esperto", definido operacionalmente pelos sujeitos entrevistados como "driblar obstáculos para conseguir o que quero", 2) "sonhador' (ter sempre uma visão otimista do futuro), 3) "vaidade" (preocupação e cuidado com minha aparência) e 4) "trabalho" (modo digno de ganhar a vida). Esse último foi sugerido pelos entrevistados como sendo característico da cultura brasileira por causa dos altos índices de desemprego e das condições nas quais o trabalho é realizado no país. Tamayo e Schwartz (1993) mostraram que, efetivamente, o valor trabalho não é visto pelos brasileiros como uma forma de auto-realização nem como a satisfação de uma necessidade pessoal, mas como um meio para garantir a subsistência da família. Além disso, os seus resultados revelaram que os quatro valores postulados como característicos da cultura brasileira se integram adequadamente na matriz motivacional, obtida pela Smallest Space Analysis, e apresentam correlações significativas com os valores integrantes do seu respectivo tipo motivacional.

\section{Objetivos}

Os objetivos da presente pesquisa foram os seguintes: 1) estabelecer, com uma amostra brasileira, a hierarquia de valores transculturais; 2) verificar se os quatro valores identificados como peculiares aos brasileiros são efetivamente considerados como valores e se integram-se adequadamente na hierarquia axiológica; 3 ) estabelecer a hierarquia de valores a partir dos coeficientes de importância dos tipos motivacionais de valores (Tamayo \& Schwartz, 1993) e 4) verificar as diferenças existentes na estrutura bidimensional e na hierarquia dos tipos motivacionais de valores de homens e mulheres, de professores de escola secundária e de estudantes universitários.

\section{Método}

\section{Amostra}

A amostra foi composta por 419 sujeitos, sendo 169 professores da escola secundária privada e 250 estudantes universitários. Duzentos sujeitos eram do sexo masculino e 219 do feminino. A idade média foi de 26 anos $(D P=7,16)$.

\section{Instrumento de medida e procedimento}

Os valores foram avaliados pela escala preparada por Schwartz (1992) para uma pesquisa multicultural. Como já foi mencionado anteriormente, ela consta de 56 valores, 30 terminais e 26 instrumentais. Os terminais foram formulados em forma de substantivos e apresentados na primeira parte do questionário; os instrumentais, expressos como formas de agir, ocuparam a segunda parte, precedidos de instruções específicas. Além disso, foram introduzidos mais quatro valores, dois terminais (trabalho e vaidade) e dois instrumentais (esperto e sonhador), postulados como sendo peculiares à cultura brasileira. Eles foram distribuídos no questionário a intervalos iguais.

A importância dos valores "como princípios que orientam a minha vida" foi avaliada por meio de uma escala de 0 a 6 . Quanto mais alto o número, mais importante é o valor para a pessoa. Além disso, foram utilizados -1 e 7 . O primeiro "significa que o valor é oposto aos princípios que orientam sua vida" e 7 "significa que o valor é de suprema importância como um princípio orientador em sua vida; geralmente, uma pessoa não possui mais do que dois desses valores". Para cada série de valores os sujeitos foram solicitados a ler todos eles e escolher, primeiro, o valor mais importante; em seguida, 
os valores opostos aos seus e, finalmente, avaliar o resto dos valores por meio da escala de 0 a 6 . Os questionários foram administrados em grupos de 5 a 10 sujeitos em condições favoráveis para esse tipo de atividade e cuidadosamente padronizadas.

\section{Resultados e Discussão}

\section{Hierarquia de valores}

A Figura 3 apresenta a hierarquia dos 60 valores para a amostra total. A escala vertical representa os graus de importância dos valores; as duas áreas por ela delimitadas foram utilizadas para colocar, à direita da escala vertical, os valores terminais e, à esquerda, os instrumentais. Cada valor foi colocado no seu lugar correspondente, de acordo com seu escore médio de importância. Como se pode observar, ao situar dessa forma os valores na escala vertical, formaramse cinco regiões, nitidamente delimitadas, delineando assim cinco níveis hierárquicos de valores (vide Tamayo, 1988).

O primeiro nível axiológico foi constituído por quatro valores terminais: "harmonia interior" (em paz comigo mesmo), "amizade verdadeira" (amigos próximos e apoiadores), "liberdade" (liberdade de ação e pensamento), "trabalho" (modo digno de ganhar a vida) e dois instrumentais: "saudável" (gozar de boa saúde física e mental) e "honesto" (ser sincero, autêntico). Esses valores podem ser considerados como os valores supremos. De fato, eles obtiveram as mais elevadas freqüências no grau 7 da escala que, como foi explicado anteriormente, significa que o valor é de suprema importância para o indivíduo.

O nível mais baixo na hierarquia foi constituído por quatro valores terminais: "riqueza" (posses materiais, dinheiro), "segurança nacional" (proteção da minha nação contra inimigos), "respeito pela tradição" (preservação de costumes vigentes há longo tempo) e "autoridade" (direito de liderar ou de mandar) e por três valores instrumentais: "audacioso" (procurar a aventura, o risco), "influente" (exercer impacto sobre pessoas e eventos) e "devoto" (apegar-me fortemente à fé religiosa). Faz-se mister mencionar que o valor "poder social" (controle sobre os outros, domínio) não foi considerado pelos sujeitos como um verdadeiro valor. O seu escore médio foi 1,49 e, por esse motivo, nem sequer foi representado na hierarquia de valores da Figura 3. Dos 60 valores da escala, ele obteve a porcentagem mais elevada de rejeição (escore -1); $26 \%$ dos sujeitos responderam que ele é "oposto aos princípios que orientam" a sua vida.

\section{Valores brasileiros}

Com relação aos quatro valores postulados como peculiares à cultura brasileira, os resultados revelaram que eles efetivamente possuem o status de valor, já que se integraram adequadamente na hierarquia de valores e se situaram em níveis relativamente elevados. Como pode ser observado na Figura 3, o "trabalho" é um dos valores supremos, ele apresentou um escore médio de 5,07, e se situa no primeiro nível da hierarquia. Tamayo e Schwartz (1993) mostraram que ele se situa na região dos valores a serviço de interesses coletivos e que sua meta motivacional é a subsistência da família.
$\mathrm{Na}$ tradição da cultura ocidental, o trabalho é considerado, basicamente, como uma necessidade pessoal cuja satisfação está associada à produtividade e ao sucesso. Teoricamente, portanto, a expectativa era de que o "trabalho" teria como meta motivacional a procura do sucesso pessoal por uma demonstração de competência. A significação dada ao valor trabalho pelos sujeitos da amostra pode ser explicada pelas condições gerais nas quais é executado o trabalho no país. Necessário se constitui, porém, salientar que, mesmo assim, o trabalho surgiu como um dos valores supremos, o qual certamente contradiz a opinião, mais ou menos generalizada, de que o brasileiro não valoriza suficientemente o trabalho. O resultado aqui observado indica que, apesar das condições indignas que acompanham a sua execução, os sujeitos revelaram ter uma motivação elevada para o trabalho, colocando-o entre os valores supremos que orientam sua vida.

Os outros três valores postulados como peculiares ocuparam posições elevadas na hierarquia, embora mais modestas que a do "trabalho", revelando, dessa forma, que eles também são princípios que orientam a vida das pessoas. "Sonhador" se situou no terceiro nível axiológico, com um escore médio de 3,75. Esse valor, que tem como

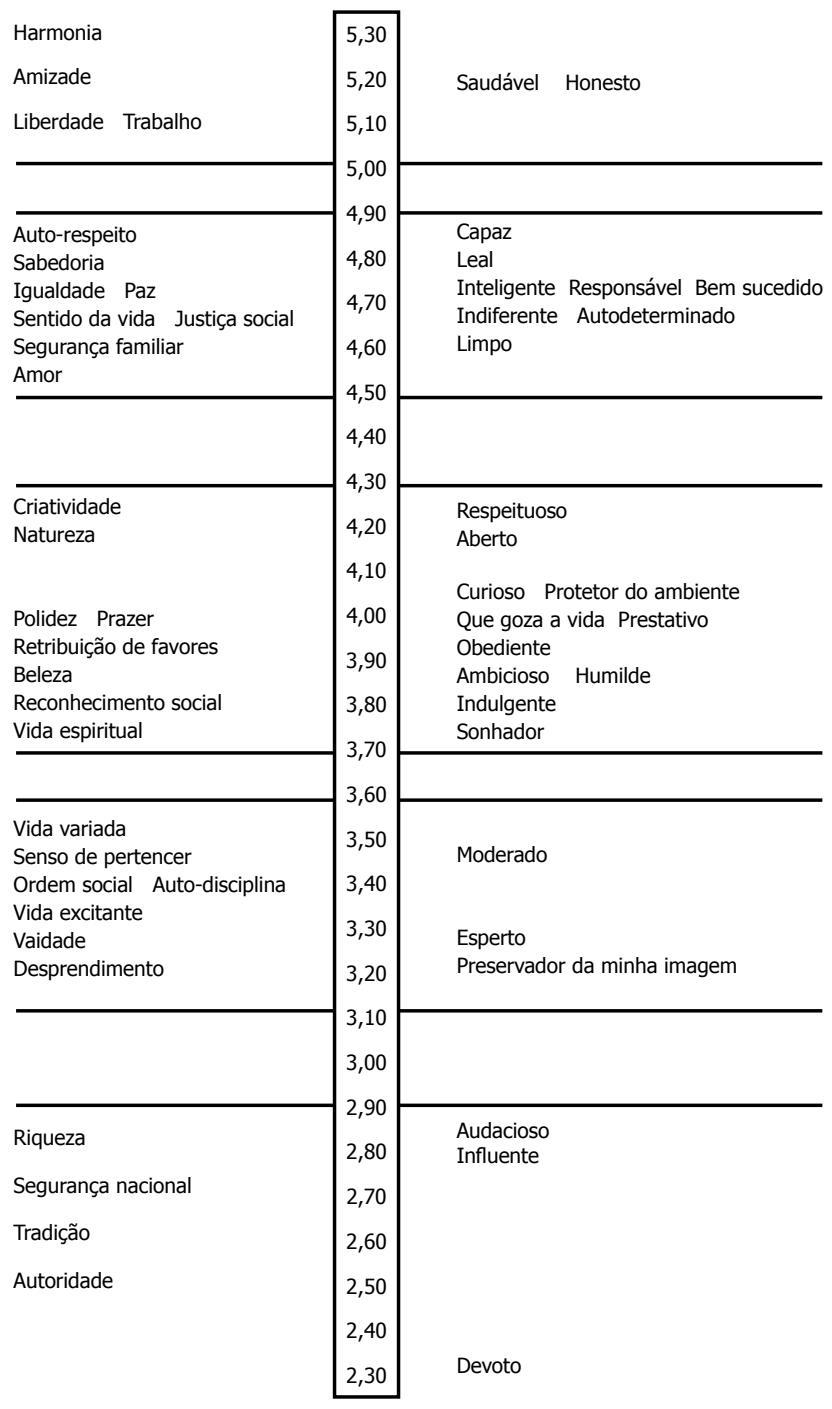

Figura 3. Hierarquia de valores. 


\section{A. Tamayo}

meta manter sempre uma visão otimista do futuro, pode estar relacionado com aquela expectativa pouco realista observada por Rodrigues (1984) em sujeitos brasileiros e por ele denominada de "otimismo ingênuo". "Vaidade" e "esperto" localizaram-se no quarto nível e apresentaram um escore médio de 3,21 e 3,20, respectivamente. Os resultados de Tamayo e Schwartz (1993) sugerem que o valor vaidade tem como meta motivacional atingir prestígio e status social a fim de obter controle ou domínio sobre os outros, e que o valor "esperto" é uma forma de auto-realização. Os quatro valores postulados como peculiares à cultura brasileira, portanto, ocuparam posições na hierarquia axiológica significativamente mais importantes que a de vários estados de existência ou modelos de comportamento desejáveis, tradicionalmente aceitos como valores, tais como "autoridade", "respeito à tradição", "segurança nacional" "riqueza", "audacioso", "influente" e "devoto".

\section{Hierarquia dos tipos motivacionais}

A hierarquia para os tipos motivacionais de valores foi estabelecida para a amostra total a partir dos escores médios de importância (Tabela 1).

Tabela 1. Escore de importância dos tipos motivacionais e desvio padrão para a amostra total.

\begin{tabular}{lcc}
\hline Tipos motivacionais & Escore & DP \\
\hline Autodeterminação & 4,61 & 1,03 \\
Benevolência & 4,49 & 0,92 \\
Filantropia & 4,49 & 0,90 \\
Auto-realização & 4,29 & 1,07 \\
Hedonismo & 3,96 & 1,50 \\
Conformidade & 3,87 & 1,15 \\
Segurança & 3,83 & 1,02 \\
Estimulação & 3,31 & 1,20 \\
Tradição & 3,14 & 1,17 \\
Poder & 2,79 & 1,02 \\
\hline
\end{tabular}

Os valores relativos à Autodeterminação ocuparam o primeiro lugar; a sua meta motivacional é a procura de liberdade de pensamento, de ação e de opção. Os valores característicos desse tipo motivacional são a criatividade, a liberdade, o auto-respeito, a independência, a curiosidade e a capacidade para escolher as suas próprias metas. No segundo lugar da hierarquia situaram-se os tipos motivacionais Benevolência e Filantropia; o primeiro compreende valores tais como "amor', "trabalho", "amizade", e tem como meta o bem-estar da família e das pessoas íntimas. A meta do segundo é o bem-estar de todos e é constituído por valores como "justiça social", "igualdade", "paz", "união com a natureza", "protetor do ambiente", etc. Os valores integrantes do tipo motivacional Auto-realização, cuja meta é a procura de sucesso pessoal por meio de uma demonstração de competência, ocuparam o terceiro lugar. Esse tipo motivacional é constituído pelos valores "bemsucedido", “capaz”, "ambicioso", "inteligente”, "esperto” e "influente". O tipo motivacional Hedonismo, que tem como alvo a gratificação sensual, ocupou o quarto lugar, seguido de Conformidade, cuja meta motivacional é o controle dos impulsos a partir de normas estabelecidas pela sociedade. No sexto lugar foi observado o tipo motivacional Segurança, constituído por valores como "ordem social", "segurança familiar, "segurança nacional", "reciprocidade de favores", etc. A meta motivacional desse grupo de valores é a integridade pessoal, a integridade dos grupos de referência e dos outros em geral. Embaixo na hierarquia situaram-se os tipos motivacionais Estimulação ("uma vida variada", "uma vida excitante", "audacioso"), Tradição ("respeito pela tradição", "moderado", "ciente dos meus limites") e Poder ("poder social", "autoridade", "reconhecimento social", "riqueza").

\section{Tipos motivacionais diferenças intergrupais}

Para verificar as possíveis diferenças na hierarquia dos tipos motivacionais de valores em função do gênero e da profissão, aqui representada pelas amostras de professores de escola secundária e de estudantes universitários, foi calculada a Anova 2x2 em termos de cada um dos tipos motivacionais. Foram observadas diferenças de gênero nos seguintes tipos motivacionais: Hedonismo $[F(412,1)=9,76$; $p \leq 0,002]$, Benevolência $[F(405,1)=10,54 ; p \leq 0,001]$, Tradição $[F(408,1)=7,22 ; p \leq 0,008]$, Conformidade $[F(411,1)=3,77$; $p \leq 0,05]$, Segurança $[F(405,1)=16,52 ; p \leq 0,0011]$ e Filantropia $[F(408,1)=4,02 ; p \leq 0,04]$. No tipo motivacional Hedonismo o escore foi mais elevado para os sujeitos masculinos do que para os femininos; nos outros tipos motivacionais, as mulheres apresentaram escores mais altos do que os homens. Cabe salientar também que, no plano do tipo motivacional Estimulação, foi observada uma tendência $[F(407,1)=4,21$; $p \leq 0,07]$, sendo o escore superior para o sexo masculino do que para o feminino (Figura 4).

Numerosas pesquisas têm mostrado que os valores possuem uma orientação individualista ou coletivista, segundo estejam a serviço de interesses individuais ou coletivos (Kluckhohn, 1951; Kluckhohn \& Strodtbeck, 1961; Schwartz \& Bilsky, 1987, 1990; Schwartz, 1992; Tamayo \& Schwartz, 1993). Os resultados relativos ao impacto do gênero sobre a hierarquia de tipos motivacionais de valores observados na presente pesquisa (Figura 4), revelam que os homens dão prioridade aos valores que estão ao serviço de interesses individuais (Hedonismo e Estimulação), ao passo que as mulheres enfatizam mais os valores cuja meta motivacional visa interesses coletivos (Benevolência, Tradição, Conformidade) e mistos (Segurança e Filantropia). O perfil axiológico das mulheres é nitidamente mais alocêntrico que o dos homens; elas dão preferência às metas que estão a serviço do ingroup e da coletividade em geral. As metas individuais parecem estar subordinadas à procura da integridade e segurança da família, harmonia das relações e interdependência de todos na sociedade. Os 
tipos motivacionais de valores enfatizados pelos homens, ao contrário, manifestam uma tendência ao idiocentrismo, cujo núcleo consiste em dar prioridade às metas pessoais sobre as metas do grupo e da coletividade. O perfil axiológico do homem é caracterizado pela ênfase dada aos valores que servem o self por meio da gratificação sensual e da variedade na estimulação. Os valores relativos ao bem-estar da família e dos grupos de referência, à segurança, à tradição, à conformidade e à filantropia apresentaram coeficientes de importância inferiores aos das mulheres.

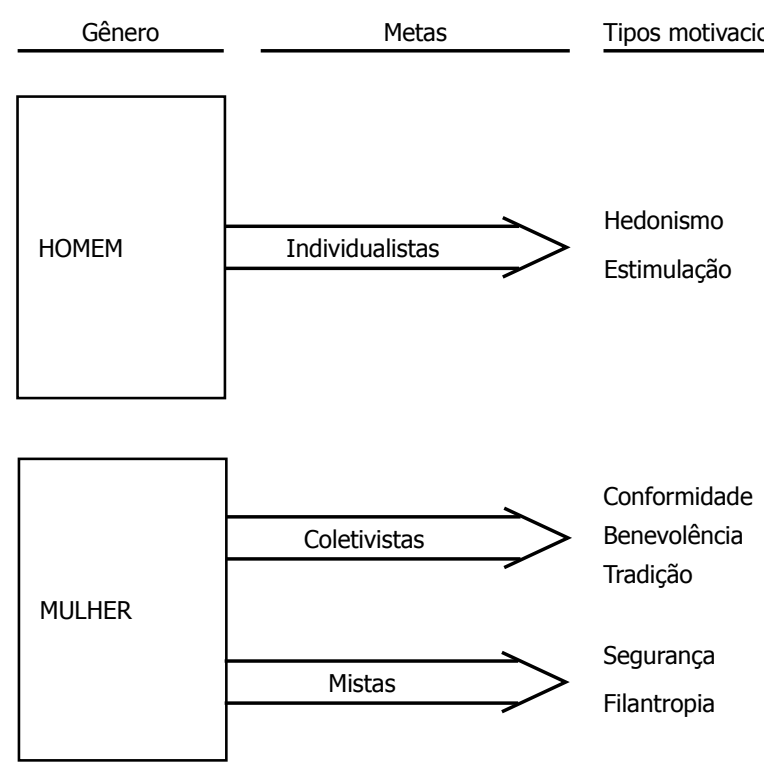

Figura 4. Diferença de gênero nos tipos motivacionais de valores.

Ao comparar o grupo de professores com o de estudantes universitários também foram observadas várias diferenças. Os estudantes apresentaram escores superiores aos dos professores nos seguintes tipos motivacionais: Autodeterminação $[F(410,1)=15,96 ; p \leq 0,001]$, Hedonismo $[F(412,1)=23,90 ; p \leq 0,001]$, Estimulação $[F(407,1)=23,11$; $p \leq 0,001]$, Auto-realização $[F(410,1)=16,78 ; p \leq 0,001] \mathrm{e}$ Filantropia $[F(408,1)=4,29 ; \mathrm{p} \leq 0,03]$. Nos tipos motivacionais Tradição $[F(408,1)=4,53 ; p \leq 0,03]$ e Conformidade $[F(411,1)=6,04 ; p \leq 0,01]$ foram observados escores mais elevados para os professores do que para os estudantes. A Figura 5 ilustra esses resultados. Interessante se constitui observar que os tipos motivacionais de valores enfatizados pelos estudantes estão todos a serviço de interesses individuais, com exceção do de Filantropia, que é misto, ao passo que os professores valorizaram mais dois tipos motivacionais coletivos, Tradição e Conformidade. Os resultados dos estudantes, portanto, revelaram uma maior tendência ao individualismo que os dos professores. Triandis e seus colegas estudaram a relação entre os valores e os escores dos sujeitos numa escala de individualismo-coletivismo. $\mathrm{O}$ grupo dos individualistas extremos deram prioridade a todos os valores de tipo individual (Triandis \& cols., 1986). O perfil axiológico dos estudantes apresenta semelhanças com o dos individualistas extremos, no sentido em que eles enfatizaram todos os tipos motivacionais de valores a serviço de interesses individuais, com exceção do Poder. $\mathrm{O}$ perfil dos estudantes difere também do individualista extremo, na importância dada pelos estudantes aos valores do tipo motivacional Filantropia.

Talvez as diferenças observadas entre os professores e os estudantes universitários possam ser explicadas pelo fator idade: a amostra de professores era constituída por profissionais, a maioria casados e com idade média de 31,27 anos, contra 22 anos para a amostra de estudantes. Obviamente, a idade está associada a outras variáveis, podendo ser vivências ou fases cruciais para a pessoa, tais como experiência de trabalho e fundação de uma família que, facilmente, levam a níveis diferentes de maturação psicológica.

\section{Estrutura bidimensional: diferenças intergrupais}

A estrutura bidimensional dos valores é constituída por quatro fatores de ordem superior, dispostos em dois eixos bipolares (Figura 2). A Anova $2 \times 2$ foi calculada no plano de cada um desses quatro fatores para verificar o impacto do gênero e da profissão (professor $v s$ estudante universitário). Foi observado um efeito principal da variável gênero sobre os fatores Auto-transcendência $[F(402,1)=5,31 ; p \leq 0,005]$ e Conservação $[F(401,1)=12,77 ; p \leq 0,0009]$, sendo nos dois casos o escore das mulheres superior ao dos homens. Esses resultados confirmam observações anteriormente realizadas com adolescentes (Tamayo, 1993). A Auto-transcendência dos interesses próprios em favor dos interesses dos outros está constituída pelos tipos motivacionais Benevolência e Filantropia, que têm como meta motivacional a preocupação com o bem-estar das pessoas íntimas e da sociedade em geral. Os resultados obtidos para os sujeitos do sexo feminino revelam, portanto, a sua tendência a transcender, mais do que os homens, as preocupações egoístas e de promover o bem-estar dos outros. Além disso, as mulheres apresentaram escores mais elevados do que os sujeitos do sexo masculino no fator Conservação, que compreende valores cuja meta motivacional é de manter o status quo. Ao manter o status quo, a pessoa está preservando a segurança e a certeza que ela oferece no relacionamento com os outros e com as instituições.

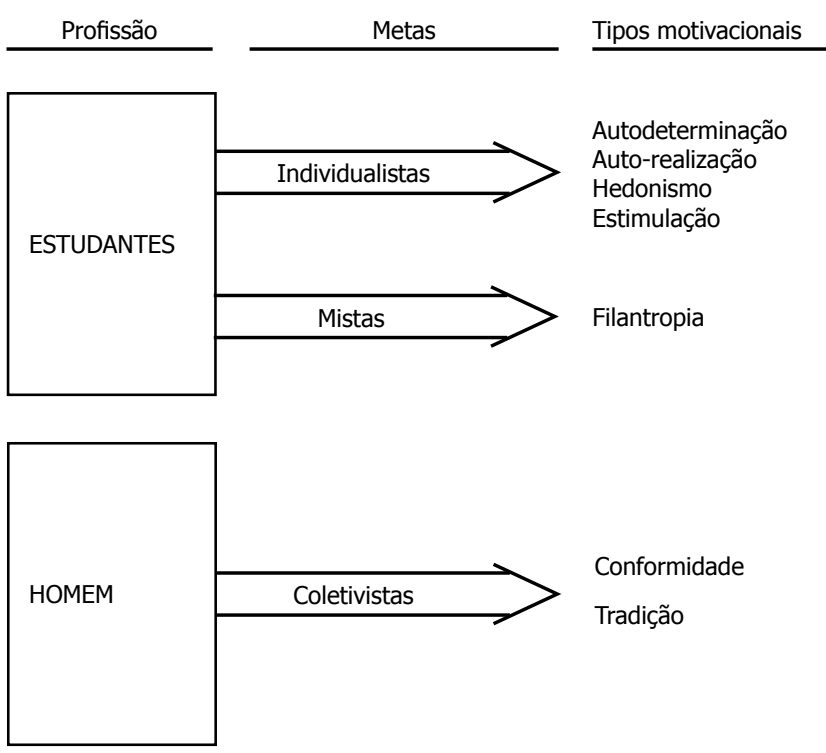

Figura 5. Diferenças entre estudantes universitários e professores nos tipos motivacionais de valores. 


\section{A. Tamayo}

Esses resultados parecem oferecer uma imagem mais realista da estrutura axiológica das mulheres do que os resultados de pesquisas anteriores. Consistentemente os pesquisadores têm observado que as mulheres, mais do que os homens, interessam-se pelos valores afetivos, estéticos e religiosos (Allport \& Vernon, 1931; Allport, Vernon, \& Lindzey, 1970; Cartwright, 1972; Walberg, 1969). Cabe lembrar também a opinião de Horney (1969), segundo a qual as mulheres supervalorizam o amor, a moral e a religião. De acordo com as observações realizadas nessa pesquisa, a "supervalorização" realizada pelas mulheres é mais em termos dos interesses da coletividade e da conservação do status quo do que em termos do amor, da moral e da religião.

Com relação à variável profissão, foi observado um efeito significativo, o nível dos fatores Auto-promoção $[F(403,1)=6,29 ; p<0,01]$ e Abertura à mudança $[F(403,1)=30,36 ; p<0,0009]$. Nos dois casos, o escore dos estudantes universitários foi superior ao dos professores, revelando que os primeiros apresentam uma maior motivação para fomentar e prosseguir os seus próprios interesses, mesmo às custas dos outros, e para seguir a direção definida pelas suas próprias idéias e emoções, mesmo se ela é incerta e insegura. Essas preferências axiológicas do estudante universitário sugerem que as suas preocupações motivacionais básicas, nesse período da vida, se focalizam em torno de si mesmo. De um lado, sua promoção, mesmo às custas dos outros, na área afetiva, social e intelectual e, do outro, sua autonomia, isso é, poder dispor de si mesmo, nas diversas situações da sua vida, de acordo com sua própria escala de valores.

\section{Conclusões}

A hierarquia de valores observada é bem estruturada, composta por cinco níveis, nitidamente delimitados. O primeiro compreende os valores supremos (harmonia interior, amizade, liberdade, trabalho, saudável e honesto) e o quinto nível aqueles que têm menos importância para os sujeitos. Entre os valores desse último nível cabe salientar, como indicadores de mudança cultural importante, o "respeito à tradição", a "autoridade" e a "segurança nacional".

Os quatro valores postulados como característicos à cultura brasileira integraram-se na hierarquia axiológica em termos de importância significativos, superiores aos de vários valores tradicionais. Com base nesse resultado e nas observações de Tamayo e Schwartz (1993), relativas à forma como esses valores se integram na matriz motivacional, podese concluir que, para as amostras analisadas, os dois estados de existência representados pelos itens "trabalho" e "vaidade" e os dois modelos de comportamento desejáveis, "esperto"e "sonhador", são efetivamente valores. Embora essa conclusão não possa ser generalizada, os resultados sugerem que os quatro valores estudados podem ser característicos da cultura brasileira. Isso não significa, porém, que eles sejam exclusivos, isso é, específicos. Um estudo com uma amostra nacional atualmente em andamento esclarecerá até que ponto esses quatro valores são verdadeiramente característicos da cultura brasileira.

Do ponto de vista motivacional, a prioridade foi para os conjuntos de valores que têm como meta a procura de autodeterminação e o bem-estar das pessoas íntimas. Do ponto de vista da dicotomia, individualismo $v s$ coletivismo, a hierarquia de tipos motivacionais é equilibrada, já que a amostra total não apresentou tendência significativa de favorecer um dos pólos em detrimento do outro.

A estrutura axiológica foi influenciada pelo gênero e a profissão dos sujeitos (professores $v s$ estudantes). As mulheres, quando comparadas com os homens, apresentaram um perfil axiológico mais voltado para a coletividade, a conservação do status quo, a autotranscendência dos interesses egoístas e a promoção do bem-estar dos outros. O perfil axiológico dos estudantes universitários foi marcado pela inclinação ao individualismo extremo, à procura da promoção de si mesmo, à tendência a seguir os seus interesses intelectuais e emocionais numa direção incerta, em oposição à segurança conferida pela tradição e o status quo.

\section{Referências}

Allport, G. W. \& Vernon, P. E. (1931). A study of values: Manual of direction. Boston: Houghton Mifflin.

Allport, G. W., Vernon, P. E. \& Lindzey, J. W. (1970). Manual, study of values. Cambridge: Houghton Mifflin.

Ball-Rokeach, S. J., Rokeach, M. \& Grube, J. W. (1984). The great American values test. Psychology Today, 18(11), 34-41.

Braithwaite, V. A. \& Law, H. G. (1985). Structure of human values. Testing the adequacy of the Rokeach value survey. Journal of Personality and Social Psychology, 49, 250-263.

Bond, M. H. (1988). Finding universal dimensions of individual variation in multicultural studies of values: The Rokeach and Chinese values surveys. Journal of Personality and Social Psychology, 55, 1009-1015.

Canter, D. (1985). Facet theory: Approaches to social research. New York: Springer-Verlag.

Cartwright, L. K. (1972). Personality differences in male and female medical students. Psychiatry and Medicine, 3, 213-218.

Chinese Culture Connection (1987). Chinese values and the search for culture-free dimension of culture. Journal of Cross-cultural Psychology, 18, 143-164.

Durkheim, E. (1954). The elementary forms of religious life. New York: Free Press.

Gorsuch, R. L. (1970). Rokeach's approach to value systems and social compassion. Review of Religious Research, 11, 139143.

Guttman, L. (1968). A general non-metric technique for finding the smallest coordinate space for a configuration of points. Psychometrica, 33, 469-506.

Hofstede, G. (1980). Culture's consequences: International differences in work-related values. Beverly-Hills: Sage.

Horney, K. (1969). La psychofogre de la femme. Paris: Payot.

Inglehart, R. (1985). Aggregate stability and individual-level flux in mass belief systems: The level of analysis paradox. American Political Science Review, 79, 97-116.

Keats, D. M. \& Keats, J. A. (1974). Review of the nature of human values by M. Rokeach. Australian Journal of Psychology, 26, 164-165.

Kitwood, T. M. \& Smithers, A. G. (1975). Measurement of human values: An appraisal of the work of Milton Rokeach. Educational Research, 17, 175-179. 
Kluckhohn, F. R. \& Strodtbeck, F. L. (1961). Variations in value orientations. Evanston: Row, Peterson.

Kluckhohn, C. (1951). Values and value-orientations in the theory of action: An exploration in definition and classification. Em T. Parsons \& E. Shils (Orgs.), Toward a general theory of action (pp. 469-506). Cambridge: Harvard University Press.

Lynn, R. (1974). Review of the nature of human values by M. Rokeach. British Journal of Psychology, 65, 453.

Munro, D. (1985). A free-format values inventory: Explorations with Zimbabwean student teachers. South African Journal of Psychology, 15, 33-41.

Pereira, C. A. A. (1986). A dimensão dos valores entre habitantes de uma cidade do interior: um estudo intracultural. Manuscrito. Uberlândia: UFU.

Rodrigues, A. (1984). Atribuição de causalidade ao sucesso e fracasso como fator mediador da reação emocional e da expectiva de comportamento Arquivos Brasileiros de Psicologia, 36, 12-15.

Rokeach, M. (1968-1969). The role of values in public opinion research. Public Opinion Quarterly, 32, 547-559.

Rokeach, M. (1971). Long-range experimental modification of values, attitudes and behavior. American Psychologist, 26, 453-459.

Rokeach, M. (1973). The nature of human values. New York: Free Press.

Rokeach, M. (1974). Change and stability or American value systems, 1968-71. Public Opinion Quarterly, 38, 232-238.

Rokeach, M. (1979). Understanding human values. New York: Free Press.
Rokeach, M. \& Ball-Rokeach, S. J. (1989). Stability and change in American value priorities, 1968-1981. American Psychologist, 44, 775-784.

Schwartz, S. H. \& Bilsky, W. (1987). Toward a universal psychological structure of human values. Journal of Personality and Social Psychology, 53, 550-562.

Schwartz, S. H. \& Bilsky, W. (1990). Toward a theory of the universal content and structure of values: Extensions and cross-cultural replications. Journal of Personality and Social Psychology, 58, 878-891.

Schwartz, S. H. (1992). Universals in the content and structure of values: Theoretical advances and empirical tests in 20 countries. Em M. Zanna (Org.), Advances in experimental social psychology, Vol. 25 (pp. 1-64). San Diego: Academic Press.

Tamayo, A. (1988). Influência do sexo e da idade sobre o sistema de valores. Arquivos Brasileiros de Psicologia, 38, 91-104.

Tamayo, A. (1993). Valores de los adolescentes: ejes motivacionales. Acta Psiquiátrica y Psicológica de América Latina, 39, 140 151.

Tamayo, A. \& Schwartz, S. H. (1993). Estrutura motivacional dos valores. Psicologia: Teoria e Pesquisa, 9, 329-348.

Triandis, H. C., Bontempo, R., Betancourt, H., Bond, M., Leung, K., Brenes, A., Georgas, J., Hui, C. H., Marin, G., Setiadi, B., Sinha, J. B. P., Verna, J., Spangenbert, J., Touzard, H. \& de Montmollin, G. (1986). The measurement of ethic aspects of individualism and collectivism across cultures. Australian Journal of Psychology, 38, 257-267.

Walberg, H. J. (1969). Physics, feminity and creativity. Developmental Psychology, 1, 47-54. 